\title{
Synthesis, Molecular Docking and Antimicrobial Evaluation of New Tetrahydrobenzothienopyrimidine Derivatives
}

\author{
Neetu Chopra, Kiranpreet kaur, Sanjeev Kumar \\ Department of Pharmaceutical Chemistry, \\ Rajendra Institute of Technology and Sciences (RITS), Sirsa, Haryana, India
}

\section{ABSTRACT}

A series of novel derivatives of Various thienopyrimidine derivatives are reported to Tetrahydrobenzothienopyrimidine hydrazone were possess broad spectrum of biological activities such synthesized and product structure was elucidated by 1NMR, C13NMR and mass spectroscopy. The synthesized compounds were evaluated against fungal and bacterial strains. The synthesized compounds showed significant antibacterial activity against Staphylococcus aureus MTCC 96, Staphylococcus pyrogenes MTCC 442, and Escherichia coli MTCC 443, Pseudomonas aeruginosa MTCC 1688 and against fungal strains Candida albicans MTCC 227, Aspergillus niger MTCC 282, Aspergillus clavatus MTCC 1323. Some derivatives showed promising result against gram positive, gram negative bacterial and fungal strains than standard drug ampicillin and grieseofulvin. In- silico molecular docking studies of the synthesized compounds was done by using GRIP batch docking method of Vlife MDS 3.0 software to study their observed activity which showed a significant correlation between the binding score and biological activity for synthesized compounds.

Keyword: $\quad$ Tetrahydrobenzothienopyrimidines, Ampicillin, Griesofulvin, NMR, Pseudomonas aeruginosa, Escherichia coli, Candida albicans.

\section{INTRODUCTION}

Tetrahydrobenzothienopyrimidines are important class of heterocyclic compounds possessing significant biological activities and interesting chemical features. Thienopyrimidines occupy a special position among the sulphur containing compounds. Along with some other pyrimidine systems containing an annulated five membered hetero aromatic ring, Thienopyrimidines are structural analogs of biogenic purines and can be considered as potential nucleic acid antimetabolites. as antiviral, anticancer, anti-inflammatory, antimicrobial, antihistaminic, antipyretics, antianaphylactic, anticonvulsant and immunostimulant properties.[1-3] Besides many thienopyrimidines compounds also exhibited analgesic, neurotropic, molluscicidal and larvicidal activities[4]. Tetrahydrobenzothienopyrimidine derivatives have been reported a valuable drug for many years exhibiting Trypanoside, AntiTuberculosis, Leishmanicidal and Cytotoxic Activities [5-6]. They are relatively easy obtainable, less toxic, and shows promising biological activities. The chemical structure modification of these compounds was carried out mainly by introduction of new substituents at hydrazinyl group. Substitution of N- substituted indole aldehyde; other substituted benzaldehydes like dimethyl benzaldehyde, mhydroxy aldehyde etc. and $\mathrm{O}$-alkylated vanillin, such modification are expected to change not only potency but also the biological activities [7]. The substituted derivatives exhibit promising antimicrobial activity [8]. Various substituted tetrahydrobenzothienopyrimidines showed antimicrobial activity [9]. Analgesic and antiinflammatory activity [10], anticancer activity [11], anticonvulsant activity [12], antiparasitic activity [13] summarized recently in the review articles. Our strategy for introducing various substituted aldehyde at hydrazinyl group of the tetrahydrobenzothienopyrimidine to form hydrazones. We found that derivative compounds exhibit promising antimicrobial activity against gram positive bacteria, gram negative bacterial and fungal strain. We found that some compounds showed less MIC value compared to standard drug ampicillin and 
grisofulvin. The compounds were elucidated by ${ }^{1} \mathrm{NMR}$ and $\mathrm{C}^{13} \mathrm{NMR}$ and mass spectroscopy. In this work, we discuss the synthesis, molecular docking and the structure elucidation of the various hydrazones of tetrahydrobenzothienopyrimidines and their antimicrobial activities.

\section{Material and methods:}

\section{Synthesis of compounds}

Melting points were determined in open capillary tubes and ${ }^{1} \mathrm{H}$; ${ }^{13} \mathrm{C}$ NMR spectra of the compounds were recorded using $\mathrm{CDCl}_{3}$ and $\mathrm{DMSO}-\mathrm{d}_{6}$ as solvent in all cases. Tetramethylsilane (TMS) was used as an internal standard. Chemical shifts are recorded in parts per million (ppm, $\delta$ ) and the signals are described as s (singlet), d (doublet), t (triplet), $\mathrm{q}$ (quartet) and $\mathrm{m}$ (multiplet). The value of coupling constant $(J)$ is given in Hz. The IR spectra of the synthesized compounds were recorded on a Fourier Transform IR spectrometer (Nicolet 380 FT-IR) in the range of $400-4000$ using $\mathrm{KBr}$ pellets and the values of $v_{\max }$ are reported in $\mathrm{cm}^{-1}$. Thin layer chromatography (TLC) was done by using precoated silica plates (Merk, Silica gel $60 \mathrm{~F}_{254}$ ) for analysis. The spot were visualized either by exposing the developed and dried plates to iodine vapors or in UV chamber (254 and $365 \mathrm{~nm}$ ). Synthesis and purity of all new compounds was accomplished by monitoring with TLC. Colum chromatography was also performed with silica having (60-120 mesh). All the compounds were evaluated for antibacterial and antifungal activity by using broth micro dilution method.

\section{Synthesis of aminothiophene ester (4)}

Cyanoacetic acid ethyl ester $(0.23 \mathrm{moL})$, sulphur $(0.23 \mathrm{moL})$, cyclohexanone $(0.23 \mathrm{moL})$ were dissolved in absolute ethanol and the mixture was stirred at room temperature for 5 minutes. After that diethyl amine (1.2 eqiv.) was added drop wise. The reaction mixture was refluxed at $40^{\circ} \mathrm{C}$ for 3-4 hrs. Reaction was monitored by T.L.C. After the completion of reaction solid product was filtered and washed with $20 \%$ aqueous ethanol to get pure product. [14]. Melting point- $150-152^{\circ} \mathrm{C}$, yield $78 \%$, Rf 0.68.IR (KBr) vmax (cm-1): $3405(\mathrm{~N}-\mathrm{H}), 3168$ (aromatic C-H), 2985 (aliphatic C-H), $1647(\mathrm{C}=\mathrm{O})$, $1596(\mathrm{C}=\mathrm{C}), 1276(\mathrm{C}-\mathrm{O})$.
Synthesis of 4-hydroxy-5, 6-tetramethylenethieno [2, 3-d] pyrimidine (5)

2-Amino-4, 5, 6, 7-tetrahydro-3ethoxycarbonylbenzothiophene, $(4,43.3 \mathrm{gm})$ was suspended in form amide (400 mL) and refluxed for 6 hrs. After cooling to room temperature, the dark solution was placed inside a refrigerator overnight, and the dark brown needles of 4-hydroxy-5,6tetramethylenethieno[2,3-d] pyrimidine (5) were filtered and washed with a cold solution of $40 \% \mathrm{EtOH}$ in $\mathrm{H}_{2}$ O.m.p $170-172^{\circ} \mathrm{C}$, IR $(\mathrm{KBr}) \mathrm{V}_{\max }\left(\mathrm{cm}^{-1}\right): 3468$ (N-H), 3010 (aromatic C-H), 2938 (aliphatic C-H), $1658(\mathrm{C}=\mathrm{O}), 1589(\mathrm{C}=\mathrm{N}), 1544(\mathrm{C}=\mathrm{C}), 1170(\mathrm{CN})$

\section{Formation of 4-chloro-5, 6, 7, 8-tetrahydro-} benzothieno $[2,3-d]$ pyrimidine $(6)$

4-hydroxy-5, 6, 7, 8-tetramethylenethieno [2, 3d]pyrimidine $(5,37.3 \mathrm{gm})$ was suspended in $\mathrm{POCl}_{3}$ (400 $\mathrm{mL})$ and refluxed for $3 \mathrm{hrs}$. After cooling to room temperature, the excess of $\mathrm{POCl}_{3}$ was removed under reduced pressure, and then $300 \mathrm{~mL}$ of $\mathrm{CHCl}_{3}$ were added and the homogeneous solution was poured over ice water $(500 \mathrm{~mL})$. The resulting bilayer mixture was separated and the aqueous layer was adjusted to $\mathrm{pH} 8$ using $\mathrm{NH}_{4} \mathrm{OH}$ (conc.) and then extracted with $\mathrm{CHCl}_{3}$. The organic fractions were pooled and dried over anhydrous $\mathrm{Na}_{2} \mathrm{SO}_{4}$. The $\mathrm{CHCl}_{3}$ was removed under reduced pressure and the remaining yellow solid was re-dissolved in hot $\mathrm{MeOH}$. Afterwards, the solution was allowed to cool to room temperature and placed inside a refrigerator overnight. The precipitated white crystals of 4-chloro5,6,7,8-tetrahydro-[1]benzothieno[2,3-d]pyrimidine

(6) was filtered, washed with a cold solution of $80 \%$ EtOH in $\mathrm{H}_{2} \mathrm{O}$ and dried overnight under vacuum to get the required product in good yield and purity. M.p.150-152 ${ }^{\circ} \mathrm{C}$ IR (KBr) vmax (cm-1): 3207(N- H), 2937 (aromatic C-H) 2795 (aliphatic C-H), $1624(\mathrm{C}=\mathrm{N}), 1506(\mathrm{C}=\mathrm{C}), 782(\mathrm{C}-\mathrm{Cl})$.

Synthesis of hydrazinyl-5, 6, 7, 8-tetrahydro[1]benzothieno[ 2,3-d]pyrimidine (7)

To a solution of 4-chloro-5,6,7,8-tetrahydro[1]benzothieno[2,3-d]pyrimidine $(6,21.9 \mathrm{gm})$ in methanol $(400 \mathrm{~mL}), 80 \%$ hydrazine hydrate $(16 \mathrm{~mL})$ was added and the solution was refluxed for $2 \mathrm{hrs}$; immediately after, hydrazine $8 \mathrm{~mL}(99 \%)$ was added to complete the reaction and the reaction mixture was allowed to reflux for another $1 \mathrm{hr}$. After cooling to room temperature, the solution was placed inside the refrigerator overnight. The resulting yellow precipitate of 4-hydrazinyl-5, 6, 7, 8-tetrhydro 
[1] benzothieno[2,3-d]pyrimidine (7) was filtered and washed with a cold solution of $40 \% \mathrm{EtOH}$ in $\mathrm{H}_{2} \mathrm{O}$.m.p $180-182^{\circ} \mathrm{C}$. IR (KBr) vmax (cm-1): 3308(N-H), 2938 (aromatic C-H), 2835 (aliphatic C-H), 1605(C=N), $1536(\mathrm{C}=\mathrm{C}), 1 \mathrm{H}$ NMR $(300 \mathrm{MHz}$, DMSO- d6): $\delta$ (ppm) 8.35 (s, 1H, N-H); 7.89 (bs, 1H, pyrimidinyl); 4.60 (brs, 2H, NH2); 2.98 (d, J = $1.5 \mathrm{~Hz}, 2 \mathrm{H}$ ); 2.81 (d, $\mathrm{J}=2.5 \mathrm{~Hz}, 2 \mathrm{H}) ; 1.80(\mathrm{t}, \mathrm{J}=2.5 \mathrm{~Hz}, 4 \mathrm{H})$.

Synthesis of 5, 6, 7, 8-tetrahydrobenzothieno [2, 3d] pyrimidin-4-yl) hydrazine

Derivatives (8a-n)

To a solution of hydrazinyl-5,6,7,8-tetrahydro[1]benzothieno[ 2,3-d]pyrimidine (7, 1gm, $1 \mathrm{mmoL})$ in absolute ethanol $(10 \mathrm{~mL})$ was added various aldehydes ( $1 \mathrm{mmoL})$ followed by the addition of few drops of glacial acetic acid. The reaction mixture was then refluxed for 5 to $48 \mathrm{hrs}$. After cooling to room temperature reaction mixture was placed overnight inside the refrigerator and the crude solid product precipitated out was filtered, washed with a solution of cold $80 \% \mathrm{EtOH}$ in $\mathrm{H}_{2} \mathrm{O}$ and dried. The crude product was then recrystallized from absolute ethanol to get the pure titled compounds. In some case coloum chromatography was done to get the titled compounds in good yield and purity.

2-(5, 6, 7, 8-tetrahydro [1] benzothieno $[2,3 \mathrm{~d}]$ pyrimidin-4-yl) hydrazone-4-N, Ndimethyl benzaldehyde (8a) IR (KBr) $v_{\max }\left(\mathrm{cm}^{-1}\right)$ :) $3276(\mathrm{~N}-$ $\mathrm{H}), 2916$ (aromatic C-H), $1599(\mathrm{C}=\mathrm{N}), 1512(\mathrm{C}=\mathrm{C})$.

${ }^{1} \mathrm{H}$ NMR (300MHz, DMSO- $\left.\mathrm{d}_{6}\right): \delta(\mathrm{ppm}) 11.62(\mathrm{~s}$, $1 \mathrm{H}, \mathrm{NH}) ; 8.25$ (s, $1 \mathrm{H}$, pyrimidinyl); 7.75-7.73 (m, 3H, $=\mathrm{CH} \& 2 \mathrm{Ar}-\mathrm{H}) ; 6.75-6.72(\mathrm{~d}, J=8.4 \mathrm{~Hz}, 2 \mathrm{H}, \mathrm{Ar}-\mathrm{H})$; 2.97 (bs, $8 \mathrm{H}, 2 \times \mathrm{CH}_{3}$ and $\mathrm{CH}_{2}$ ); 2.73 (bs, $2 \mathrm{H}, \mathrm{CH}_{2}$ ); 1.78 (bs, $\left.4 \mathrm{H}, 2 \times \mathrm{CH}_{2}\right)$.

${ }^{13} \mathrm{C}$ NMR (300 MHz, DMSO- $\left.d_{6}\right): \delta(\mathrm{ppm}) 156.84\left(\mathrm{C}_{4}\right.$ of pyrimidinyl), 153.97 ( $\mathrm{C}_{2}$ of pyrimidinyl $), 151.82$ $\left(\underline{\mathrm{C}}-\mathrm{N}\left(\mathrm{CH}_{3}\right)_{2}\right), 148.01$ (S-C-N), $144.44 \quad(\underline{\mathrm{C}}=\mathrm{N}-\mathrm{NH})$, 132.25, 129.56, 128.60, 123.41, 119.47, 112.06, 39.99 [2드 of $\left.\mathrm{N}\left(\mathrm{CH}_{3}\right)_{2}\right], 27.00\left(\underline{\mathrm{C}}_{8}\right), 25.11\left(\underline{\mathrm{C}}_{5}\right), 22.89\left(\mathrm{C}_{7}\right)$, $22.50\left(\mathrm{C}_{6}\right)$.

2-(5, 6, 7, 8-tetrahydro [1]benzothieno [2,3-d] pyrimidin-4-yl) hydrazone-3-bromobenzaldehyde(8b) IR (KBr) vmax (cm-1): 3271(N$\mathrm{H}$, 2934 (aromatic C-H), 2851 (aliphatic C-H), $1601(\mathrm{C}=\mathrm{N}), 1513(\mathrm{C}=\mathrm{C})$

${ }^{1} \mathrm{H}$ NMR (300MHz, DMSO- d6): $\delta$ (ppm) 11.91 (s, $1 \mathrm{H}, \mathrm{NH}) ; 8.36(\mathrm{~s}, 1 \mathrm{H}$, pyrimidinyl); $8.26(\mathrm{~s}, 1 \mathrm{H}$,
$=\mathrm{CH})$; 7.85-7.81 (m , 2H, Ar-H); 7.58-7.55 (dd, J = 7.8 and $8.1 \mathrm{~Hz}, 1 \mathrm{H}, \mathrm{Ar}-\mathrm{H}) ; 7.40-7.35(\mathrm{~d}, \mathrm{~J}=7.8 \mathrm{~Hz}$, $1 \mathrm{H}, \mathrm{Ar}-\mathrm{H}$ ); 2.97 (brs, 2H, CH2); 2.73 (brs, 2H, CH2); 1.78 (brs, $4 \mathrm{H}, 2 \times \mathrm{CH} 2$ )

${ }^{13} \mathrm{C}$ NMR (300 MHz, DMSO- $\left.d_{6}\right): \delta(\mathrm{ppm}) 154.34\left(\mathrm{C}_{4}\right.$ of pyrimidinyl), 152.37 ( $\mathrm{C}_{2}$ of pyrimidinyl), 151.52 ( $\underline{\mathrm{C}}-\mathrm{Br}), 148.31$ (S-C-N), 144.24 ( $\underline{\mathrm{C}}=\mathrm{N}-\mathrm{NH}), 134.35$, $132.46,130.60,122.41,118.27,113.26,26.90\left(\underline{\mathrm{C}}_{8}\right)$, $25.24\left(\underline{\mathrm{C}}_{5}\right), 22.69\left(\mathrm{C}_{7}\right), 22.50\left(\mathrm{C}_{6}\right)$.

2-(5, 6, 7, 8-tetrahydro [1] benzothieno [2,3-d] pyrimidin-4-yl) hydrazone-3-nitro-benzaldehyde (8c) IR (KBr) vmax (cm-1): $3423(\mathrm{~N}-\mathrm{H}), 2926$ (aromatic C-H), 2852 (aliphatic C-H), $1588(\mathrm{C}=\mathrm{N})$, $1508(\mathrm{C}=\mathrm{C}), 1554 \& 1344(\mathrm{NO} 2)$.

${ }^{1} \mathrm{H}$ NMR (300MHz, DMSO- d6): $\delta$ (ppm) 11.56 (bs, $1 \mathrm{H}, \mathrm{NH}) ; 8.67$ (s, $1 \mathrm{H}, \mathrm{Ar}-\mathrm{H}) ; 8.43$ (s, 1H, pyrimidinyl); 8.29- $8.23(\mathrm{~m}, 1 \mathrm{H}, \mathrm{Ar}-\mathrm{H}) ; 8.13-8.11$ (d, $\mathrm{J}=7.8 \mathrm{~Hz}, 1 \mathrm{H}, \mathrm{Ar}-\mathrm{H})$; 7.80-7.78 (m, 1H, =CH); 7.66$7.61(\mathrm{t}, \mathrm{J}=7.8 \mathrm{~Hz}, 1 \mathrm{H}, \mathrm{Ar}-\mathrm{H}) ; 2.91\left(\mathrm{bs}, 2 \mathrm{H}, \mathrm{CH}_{2}\right)$; 2.78 (bs, $\left.2 \mathrm{H}, \mathrm{CH}_{2}\right) ; 1.70$ (bs, $4 \mathrm{H}, 2 \times \mathrm{CH}_{2}$ ).

${ }^{13} \mathrm{C}$ NMR (300 MHz, DMSO-d6): $\delta$ (ppm): 158.23 ( $\mathrm{C}_{4}$ of pyrimidinyl), 150.97 ( $\mathrm{C}_{2}$ of pyrimidinyl $)$, $150.25\left(\mathrm{C}-\mathrm{NO}_{2}\right), 148.67(\mathrm{~S}-\mathrm{C}-\mathrm{N}), 144.13(\mathrm{C}=\mathrm{N}-\mathrm{NH})$, $137.82,134.24,132.96,131.23,130.32,124.02$, $121.89,119.24,26.96\left(\mathrm{C}_{8}\right), 25.14\left(\mathrm{C}_{5}\right), 22.73\left(\mathrm{C}_{7}\right)$ , $22.42\left(\mathrm{C}_{6}\right)$

2-(5, 6, 7, 8-tetrahydro [1]benzothieno [2,3-d] pyrimidin-4-yl) hydrazone-3-hydroxybenzaldehyde ( $8 \mathrm{~d})$

IR $(\mathrm{KBr}) \mathrm{V}_{\max }\left(\mathrm{cm}^{-1}\right)$ : $3444(\mathrm{O}-\mathrm{H}), 2929$ (aromatic C$\mathrm{H}), 2866$ (aliphatic $\mathrm{C}-\mathrm{H}), 1572(\mathrm{C}=\mathrm{N}), 1506(\mathrm{C}=\mathrm{C})$.

${ }^{1} \mathrm{H}$ NMR (300MHz, DMSO- $\left.\mathrm{d}_{6}\right): \delta$ (ppm) 11.76 (bs, $1 \mathrm{H}, \mathrm{NH}) ; 9.49(\mathrm{~s}, 1 \mathrm{H}, \mathrm{OH}) ; 8.29$ (s, 1H, pyrimidinyl); 7.77-7.76 (m, 1H, =CH); 7.36-7.32 (m, 2H, Ar-H); 7.24-7.19 (m, 1H, Ar-H); 6.83-6.80 (d, $J=7.5 \mathrm{~Hz}$, $1 \mathrm{H}, \mathrm{Ar}-\mathrm{H}) ; 2.98$ (bs, 2H, $\left.\mathrm{CH}_{2}\right) ; 2.73$ (bs, $2 \mathrm{H}, \mathrm{CH}_{2}$ ); 1.82-1.78 (bs, $4 \mathrm{H}, 2 \times \mathrm{CH}_{2}$ ).

${ }^{13} \mathrm{C}$ NMR (300 MHz, DMSO- $\left.d_{6}\right): \delta(\mathrm{ppm}): 157.43$ ( $\mathrm{C}_{4}$ of pyrimidinyl $), 153.87\left(\mathrm{C}_{2}\right.$ of pyrimidinyl $)$, 152.35 ( $\underline{\mathrm{C}}-\mathrm{OH}), 147.23(\mathrm{~S}-\underline{\mathrm{C}}-\mathrm{N}), 144.33(\underline{\mathrm{C}}=\mathrm{N}-\mathrm{NH})$, $137.52,135.42,133.42,132.56,130.60,124.83$, 121.69, 118.54, $26.74\left(\underline{\mathrm{C}}_{8}\right)$, $25.26\left(\underline{\mathrm{C}}_{5}\right), 22.90\left(\underline{\mathrm{C}}_{7}\right)$ ,22.32 ( $\left.\underline{\mathrm{C}}_{6}\right)$

2-(5, 6, 7, 8-tetrahydro [1]benzothieno $[2,3-$ d]pyrimidin-4-yl) hydrazone-3-ethoxy-4hydroxybenzaldehyde (8e) IR (KBr) vmax (cm-1): 
International Journal of Trend in Scientific Research and Development (IJTSRD) ISSN: 2456-6470

$3416(\mathrm{O}-\mathrm{H}) ; 2929$ (aliphatic C-H), $1584(\mathrm{C}=\mathrm{N}) ; 1507$ $(\mathrm{C}=\mathrm{C})$.

${ }^{1} \mathrm{H}$ NMR (300MHz, DMSO- d6): $\delta$ (ppm) 11.64 (s, $1 \mathrm{H}, \mathrm{NH}) ; 9.34$ (s, 1H, OH); 8.26 (s, 1H, pyrimidinyl); $7.75(\mathrm{~s}, 1 \mathrm{H},=\mathrm{CH}) ; 7.56(\mathrm{~s}, 1 \mathrm{H}, \mathrm{Ar}-\mathrm{H}) ; 7.28-7.25(\mathrm{~d}, \mathrm{~J}$ $=8.1 \mathrm{~Hz}, 1 \mathrm{H}, \mathrm{Ar}-\mathrm{H}) ; 6.83-6.80(\mathrm{~d}, \mathrm{~J}=8.1 \mathrm{~Hz}, 1 \mathrm{H}, \mathrm{Ar}-$ $\mathrm{H})$; 4.14-4.07 (q, J = 6.9 Hz, 2H, $\left.\mathrm{CH}_{2}-\mathrm{CH}_{3}\right), 2.96$ (bs, $2 \mathrm{H}, \mathrm{CH}_{2}$ ); 2.73 (bs, $2 \mathrm{H}, \mathrm{CH}_{2}$ ); 1.77 (bs, $4 \mathrm{H}, 2 \times \mathrm{CH}_{2}$ ); $1.07-1.02\left(\mathrm{t}, \mathrm{J}=6.9 \mathrm{~Hz}, 3 \mathrm{H}, \mathrm{CH}_{2}-\mathrm{CH}_{3}\right)$.

${ }^{13} \mathrm{C}$ NMR (300 MHz, DMSO- $\left.d_{6}\right): \delta(\mathrm{ppm}): 157.32$ ( $\mathrm{C}_{4}$ of pyrimidinyl), 153.67 ( $\mathrm{C}_{2}$ of pyrimidinyl), $152.45\left(\underline{\mathrm{C}}-\mathrm{OC}_{2} \mathrm{H}_{5}\right), 151.34(\underline{\mathrm{C}}-\mathrm{OH}), 147.60(\mathrm{~S}-\underline{\mathrm{C}}-\mathrm{N})$, $144.33(\underline{\mathrm{C}}=\mathrm{N}-\mathrm{NH}), 136.32,134.62,131.02,129.06$, $124.23,119.60,114.34,26.64\left(\underline{\mathrm{C}}_{8}\right), 25.16\left(\underline{\mathrm{C}}_{5}\right), 22.60$ $\left(\underline{\mathrm{C}}_{7}\right), 22.14\left(\underline{\mathrm{C}}_{6}\right)$

2-(5, 6, 7, 8-tetrahydro [1]benzothieno $[2,3-$ d]pyrimidin-4-yl) hydrazone-2,5-dimethoxy

benzaldehyde (8f): IR (KBr) vmax (cm-1): $3408(\mathrm{~N}$ H), 2931 (aromatic C-H), 2850 (aliphatic C-H), 1571 $(\mathrm{C}=\mathrm{N}), 1505(\mathrm{C}=\mathrm{C})$.

${ }^{1} \mathrm{H}$ NMR (300MHz, DMSO-d6): $\delta$ (ppm) 11.79 (bs, $1 \mathrm{H}, \mathrm{NH}) ; 8.63(\mathrm{~s}, 1 \mathrm{H}$, pyrimidinyl); 7.81-7.77 $(\mathrm{m}, 2 \mathrm{H}$, $=\mathrm{CH}$ and $\mathrm{Ar}-\mathrm{H}) ; 7.06-6.96(\mathrm{~m}, 2 \mathrm{H}, \mathrm{Ar}-\mathrm{H}) ; 3.81-3.76$ $\left(\mathrm{m}, 6 \mathrm{H}, 2 \times \mathrm{OCH}_{3}\right) ; 2.97$ (bs, $\left.2 \mathrm{H}, \mathrm{CH}_{2}\right) ; 2.74$ (bs, $2 \mathrm{H}$, $\left.\mathrm{CH}_{2}\right) ; 1.77$ (bs, $4 \mathrm{H}, 2 \times \mathrm{CH}_{2}$ ).

${ }^{13} \mathrm{C}$ NMR (300 MHz, DMSO-d6): $\delta$ (ppm): 157.67 ( $\mathrm{C}_{4}$ of pyrimidinyl), $153.74\left(\mathrm{C}_{2}\right.$ of pyrimidinyl), $152.81\left(\mathrm{C}-\mathrm{OCH}_{3}\right), 149.29\left(\mathrm{C}-\mathrm{OCH}_{3}\right), 148.38(\mathrm{~S}-\mathrm{C}-\mathrm{N})$, $144.28(\mathrm{C}=\mathrm{N}-\mathrm{NH}), 132.70,131.35,124.41,119.34$, 116.88, 113.31, 112.11, $56.57\left(\mathrm{OCH}_{3}\right), 56.11\left(\mathrm{OCH}_{3}\right)$, $27.10\left(\mathrm{C}_{8}\right), 25.13\left(\mathrm{C}_{5}\right), 22.85\left(\mathrm{C}_{7}\right), 22.48\left(\mathrm{C}_{6}\right)$

2-(5, 6, 7, 8-tetrahydro [1] benzothieno [2, 3-d] pyrimidin-4-yl) hydrazone-3-ethoxy-4hydroxybenzaldehyde (8g) IR $(\mathrm{KBr}) \mathrm{v}_{\max }\left(\mathrm{cm}^{-1}\right)$ : 3425 (N-H), 2924 (aromatic C-H), 2836 (aliphatic C$\mathrm{H}), 1574(\mathrm{C}=\mathrm{N}), 1507(\mathrm{C}=\mathrm{C}), 1208(\mathrm{C}-\mathrm{O})$.

${ }^{1} \mathrm{H}$ NMR (300MHz, DMSO- $\mathrm{d}_{6}$ ): $\delta$ (ppm) 11.69 (bs, $1 \mathrm{H}, \mathrm{NH}) ; 8.31$ (s, 1H, pyrimidinyl); $7.83(\mathrm{~s}, 1 \mathrm{H}$, $=\mathrm{CH}) ; 7.59$ (s, 1H, Ar-H); 7.33-7.31 (m, 1H, Ar-H); $7.00-6.98$ (d, $J=8.1 \mathrm{~Hz}, 1 \mathrm{H}, \mathrm{Ar}-\mathrm{H})$; 4.08-4.03 (q, $J=$ $\left.6.9 \mathrm{~Hz}, 2 \mathrm{H}, \mathrm{CH}_{2}-\mathrm{CH}_{3}\right), 3.85$ (s, 3H, $\left.\mathrm{OCH}_{3}\right), 2.99$ (bs, $2 \mathrm{H}, \mathrm{CH}_{2}$ ); 2.75 (bs, $\left.2 \mathrm{H}, \mathrm{CH}_{2}\right) ; 1.79$ (bs, $4 \mathrm{H}, 2 \times \mathrm{CH}_{2}$ ); $1.37-1.33$ (t, $\left.J=6.9 \mathrm{~Hz}, 3 \mathrm{H}, \mathrm{CH}_{2}-\mathrm{CH}_{3}\right)$.
${ }^{13} \mathrm{C}$ NMR (300 MHz, DMSO- $\left.d_{6}\right): \delta(\mathrm{ppm}): 157.24\left(\underline{\mathrm{C}}_{4}\right.$ of pyrimidinyl), 153.34 ( $\underline{\mathrm{C}}_{2}$ of pyrimidinyl), 151.02 $\left(\underline{\mathrm{C}}-\mathrm{OC}_{2} \mathrm{H}_{5}\right), \quad 149.24 \quad\left(\underline{\mathrm{C}}-\mathrm{OCH}_{3}\right), 148.60 \quad(\mathrm{~S}-\underline{\mathrm{C}}-\mathrm{N})$, $144.35(\underline{\mathrm{C}}=\mathrm{N}-\mathrm{NH}), 137.33,135.24,132.22,129.26$, 127.64 (2C),127.20 (3C), 122.50, 119.41, 113.53, $110.81,70.37\left(-\mathrm{O}-\mathrm{CH}_{2}\right), 56.27\left(\mathrm{OC}_{3}\right), 27.03\left(\underline{\mathrm{C}}_{8}\right)$, $25.11\left(\underline{\mathrm{C}}_{5}\right), 22.87-22.68(2 \underline{\mathrm{C}})$

2-(5, 6, 7, 8-tetrahydro [1] benzothieno[2,3-d] pyrimidin-4-yl) hydrazone-4-benzyloxy-3-methoxy benzaldehyde (8h) IR (KBr) vmax (cm-1): $3324(\mathrm{~N}-$ H), 2938 (aromatic C-H), 2850 (aliphatic C-H), 1563 $(\mathrm{C}=\mathrm{N}), 1507(\mathrm{C}=\mathrm{C}), 1266(\mathrm{C}-\mathrm{O})$.

${ }^{1} \mathrm{H}$ NMR (300MHz, DMSO- d6): $\delta$ (ppm) 11.66 (bs, $1 \mathrm{H}, \mathrm{NH}) ; 8.31(\mathrm{~s}, 1 \mathrm{H}$, pyrimidinyl $) ; 7.76(\mathrm{~s}, 1 \mathrm{H},=\mathrm{CH})$; 7.63 (s, 1H, Ar-H); 7.47-7.33 (m, 6H, Ar-H); 7.11$7.07(\mathrm{~m}, 1 \mathrm{H}, \mathrm{Ar}-\mathrm{H}) ; 5.13\left(\mathrm{~s}, 2 \mathrm{H}, \mathrm{OCH}_{2}\right) ; 3.86(\mathrm{~s}, 3 \mathrm{H}$, $\left.\mathrm{OCH}_{3}\right) ; 2.97$ (bs, 2H, CH 2$) ; 2.73$ (bs, 2H, $\mathrm{CH}_{2}$ ); 1.78 (bs, $4 \mathrm{H}, 2 \times \mathrm{CH}_{2}$ ).

${ }^{13} \mathrm{C}$ NMR (300 MHz, DMSO-d6): $\delta$ (ppm): 157.33 ( $\mathrm{C}_{4}$ of pyrimidinyl), $153.44\left(\mathrm{C}_{2}\right.$ of pyrimidinyl), $150.02\left(\mathrm{C}-\mathrm{OCH}_{2}-\right), 149.74\left(\mathrm{C}-\mathrm{OCH}_{3}\right), 148.81$ (S-C-

$\mathrm{N}), \quad 144.25(\mathrm{C}=\mathrm{N}-\mathrm{NH}), \quad 137.33, \quad 132.54, \quad 131.32$, 129.06, 128.84 (2C),128.30 (3C), 122.50, 119.41, $113.53,110.81,70.37\left(-\mathrm{O}-\mathrm{CH}_{2}\right), 56.27\left(\mathrm{OCH}_{3}\right), 27.03$ $\left(\mathrm{C}_{8}\right), 25.11\left(\mathrm{C}_{5}\right), 22.87-22.48(2 \mathrm{C})$

2-(5,6,7,8-tetrahydro[1] benzothieno[2,3d]pyrimidin-4-yl)hydrazone-indolyl Carbaldehyde (8i) IR $(\mathrm{KBr}) \mathrm{V}_{\max }\left(\mathrm{cm}^{-1}\right): 3364(\mathrm{~N}-\mathrm{H}), 3161(\mathrm{~N}-\mathrm{H})$, 2929 (aromatic C-H), $1574(\mathrm{C}=\mathrm{N}), 1508(\mathrm{C}=\mathrm{C}), 1246$ (C-N).

${ }^{1} \mathrm{H}$ NMR (300MHz, DMSO- $\left.\mathrm{d}_{6}\right): \delta$ (ppm) 11.50 (bs, $1 \mathrm{H}, \mathrm{NH}) ; 11.10$ (bs, $1 \mathrm{H}, \mathrm{N}-\mathrm{H}$ indole); 8.61 (s, $1 \mathrm{H}$, pyrimidinyl); 8.43 (s, 1H, Ar-H of indole); 7.82-7.79 $(\mathrm{d}, J=8.7 \mathrm{~Hz}, 1 \mathrm{H}, \mathrm{Ar}-\mathrm{H}) ; 7.76-7.74(\mathrm{~m}, 1 \mathrm{H},=\mathrm{CH})$; 7.45-7.42 (d, $J=8.4 \mathrm{~Hz}, 1 \mathrm{H}, \operatorname{Ar}-\mathrm{H}) ; 7.23-7.14(\mathrm{~m}$, $2 \mathrm{H}, \mathrm{Ar}-\mathrm{H}$ ); 3.01 (bs, 2H, $\mathrm{CH}_{2}$ ); 2.74 (bs, $2 \mathrm{H}, \mathrm{CH}_{2}$ ); $1.80\left(\mathrm{bs}, 4 \mathrm{H}, 2 \times \mathrm{CH}_{2}\right)$

${ }^{13} \mathrm{C}$ NMR (300 MHz, DMSO- $\left.d_{6}\right): \delta$ (ppm): 157.50 ( $\underline{\mathrm{C}}_{4}$ of pyrimidinyl), $151.33 \quad\left(\underline{\mathrm{C}}_{2}\right.$ of pyrimidinyl), 147.24 (S-C-N), $144.33(\underline{\mathrm{C}}=\mathrm{N}-\mathrm{NH}), 137.43,134.65$, $132.11,131.52,124.66,123.11,121.60,118.30$, 113.07,110.27, 26.94 ( $\left.\underline{\mathrm{C}}_{8}\right), 25.12$ ( $\left.\underline{\mathrm{C}}_{5}\right), 23.05-22.52$ (2ㄷ). 
2-(5, 6, 7, 8-tetrahydro [1] benzothieno[2,3-d] pyrimidin-4-yl)hydrazone-N-methyl-indolyl

Carbaldehyde (8j) IR (KBr) $\mathrm{V}_{\max }\left(\mathrm{cm}^{-1}\right): 3340(\mathrm{~N}-\mathrm{H})$, 2934 (aromatic C-H), $1574(\mathrm{C}=\mathrm{N}), 1527(\mathrm{C}=\mathrm{C}), 1246$ (C-N).

${ }^{1} \mathrm{H}$ NMR (300MHz, DMSO- $\left.\mathrm{d}_{6}\right): \delta(\mathrm{ppm}) 11.12$ (bs, $1 \mathrm{H}, \quad \mathrm{NH}) ; \quad 8.57 \quad(\mathrm{~s}, \quad 1 \mathrm{H}, \quad$ pyrimidinyl $) ; 8.47-$ 8.41(m,1H,Ar-H), 7.79-7.75 (m, 2H, =CH \& Ar-H); 7.49-7.42 (m, 1H, Ar-H); 7.27-7.21 (m, 2H, Ar-H); 4.05(s, 3H, N-C $\left.\underline{\mathrm{H}}_{3}\right) ; 2.91$ (bs, $\left.2 \mathrm{H}, \mathrm{CH}_{2}\right) ; 2.73$ (bs, $2 \mathrm{H}$, $\left.\mathrm{CH}_{2}\right) ; 1.78$ (bs, $4 \mathrm{H}, 2 \times \mathrm{CH}_{2}$ ).

${ }^{13} \mathrm{C}$ NMR (300 MHz, DMSO- $\left.d_{6}\right): \delta$ (ppm): 156.27 ( $\underline{\mathrm{C}}_{4}$ of pyrimidinyl), $150.49 \quad\left(\underline{\mathrm{C}}_{2}\right.$ of pyrimidinyl $)$, 146.6(S-C-N), 144.63 ( $\underline{\mathrm{C}}=\mathrm{N}-\mathrm{NH}), 138.03,134.85$, $132.01,131.32,125.36,123.20,122.96,120.99$, $119.50,112.27,110.37,33.20\left(\mathrm{~N}-\underline{\mathrm{CH}}_{3}\right), 27.02\left(\underline{\mathrm{C}}_{8}\right)$, 25.06 ( $\left.\underline{\mathrm{C}}_{5}\right), 22.85-22.48$ (2ㄷ).

2-(5, 6, 7, 8-tetrahydro [1]benzothieno[2,3-d] pyrimidin-4-yl) hydrazone-N-ethyl-indolyl Carbaldehyde (8k) IR (KBr) $\mathrm{v}_{\max }\left(\mathrm{cm}^{-1}\right)$ : 3352(N-H), 2930(aliphatic $\mathrm{C}-\mathrm{H}), \quad 1575(\mathrm{C}=\mathrm{N}), 1523(\mathrm{C}=\mathrm{C})$, 1236(C-N).

${ }^{1} \mathrm{HNMR}\left(300 \mathrm{MHz}, \mathrm{DMSO}-\mathrm{d}_{6}\right): \delta$ (ppm) $11.16(\mathrm{~s}, 1 \mathrm{H}$, $\mathrm{NH}) ; 8.58$ (s, 1H, pyrimidinyl $\mathrm{H}) ; 8.49-8.45(\mathrm{~m}, 1 \mathrm{H}$, ArH); $7.89(\mathrm{~s}, 1 \mathrm{H},=\mathrm{CH}) ; 7.83-7.78(\mathrm{~m}, 1 \mathrm{H}, \mathrm{Ar}-\mathrm{H})$; 7.55-7.52 (d, $J=8.1 \mathrm{~Hz}, 1 \mathrm{H}, \mathrm{Ar}-\mathrm{H}) ; 7.29-7.19(\mathrm{~m}, 2 \mathrm{H}$, $\mathrm{Ar}-\mathrm{H}) ; 4.28-4.21$ (q, $\left.J=7.2 \mathrm{~Hz}, 2 \mathrm{H},-\mathrm{CH}_{2}-\mathrm{CH}_{3}\right) ; 3.01$ (bs, 2H, $\left.\mathrm{CH}_{2}\right) ; 2.74$ (bs, 2H, $\left.\mathrm{CH}_{2}\right) ; 1.79(\mathrm{bs}, 4 \mathrm{H}, 2 \times$ $\mathrm{CH}_{2}$ ); $1.43-1.38$ (t, $\left.J=7.2 \mathrm{~Hz}, 3 \mathrm{H},-\mathrm{CH}_{2}-\mathrm{CH}_{3}\right)$.

${ }^{13} \mathrm{C}$ NMR (300 MHz, DMSO- $\left.d_{6}\right): \delta$ (ppm): 156.72 ( $\underline{\mathrm{C}}_{4}$ of pyrimidinyl), 151.20 ( $\underline{\mathrm{C}}_{2}$ of pyrimidinyl), 146.22 (S-C-N), $144.85(\underline{\mathrm{C}}=\mathrm{N}-\mathrm{NH}), 137.63,134.65$, $131.83,131.62,124.96,123.60,122.56,121.60$, $118.50,112.32,111.27,31.32\left(\mathrm{~N}-\underline{\mathrm{C}}_{2} \mathrm{H}_{5}\right), 26.68\left(\underline{\mathrm{C}_{8}}\right)$, 25.26 ( $\left.\underline{\mathrm{C}}_{5}\right), 23.05-22.48$ (2ㅁ).

2-(5, 6, 7, 8-tetrahydro [1] benzothieno [2, 3-d] pyrimidin-4-yl) hydrazone- $\mathrm{N}$-isopropyl-indolyl Carbaldehyde (81)

IR $(\mathrm{KBr}) \mathrm{v}_{\max }\left(\mathrm{cm}^{-1}\right): 3351(\mathrm{~N}-\mathrm{H}), 2928$ (aliphatic C$\mathrm{H}), 1576(\mathrm{C}=\mathrm{N}), 1511(\mathrm{C}=\mathrm{C}), 1241(\mathrm{C}-\mathrm{N})$.

${ }^{1} \mathrm{H}$ NMR (300MHz, DMSO- $\left.\mathrm{d}_{6}\right): \delta(\mathrm{ppm}) 11.14(\mathrm{~s}$, $1 \mathrm{H}, \mathrm{NH}) ; 8.59$ (s, 1H, pyrimidinyl); 8.44- 8.42 (m, $1 \mathrm{H}, \mathrm{Ar}-\mathrm{H}), 7.98(\mathrm{~s}, 1 \mathrm{H},=\mathrm{CH}) ; 7.79-7.78$ (m, 2H, Ar$\mathrm{H}) ; 7.57-7.54(\mathrm{~d}, J=7.8 \mathrm{~Hz}, 2 \mathrm{H}, \mathrm{Ar}-\mathrm{H}) ; 7.27-7.16$ (m, 2H, Ar-H); 4.82-4.74 (m, 1H, CH); 2.99 (bs, 2H,
$\mathrm{CH}_{2}$ ); 2.73(bs, 2H, $\left.\mathrm{CH}_{2}\right) ; 1.78$ (bs, $4 \mathrm{H}, 2 \times \mathrm{CH}_{2}$ ), $1.50-1.48\left(\mathrm{~d}, J=6.6 \mathrm{~Hz}, 6 \mathrm{H}, 2 \times \underline{\mathrm{CH}}_{3}\right)$

${ }^{13} \mathrm{C}$ NMR (300 MHz, DMSO- $\left.d_{6}\right): \delta$ (ppm): 157.62 ( $\underline{\mathrm{C}}_{4}$ of pyrimidinyl), $150.40 \quad\left(\underline{\mathrm{C}}_{2}\right.$ of pyrimidinyl), $146.64(\mathrm{~S}-\underline{\mathrm{C}}-\mathrm{N}), 144.65(\underline{\mathrm{C}}=\mathrm{N}-\mathrm{NH}), 136.93,134.44$, $131.52,131.22,124.47,123.20,122.56,121.32$, $118.40,113.20,111.27,30.52\left(\mathrm{~N}-\underline{C}_{2} \mathrm{H}_{5}\right), 26.98(\underline{\mathrm{C}} 8)$,

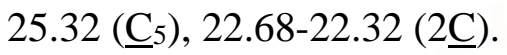

\section{2-(5, 6, 7, 8-tetrahydro [1] benzothieno [2, 3-d]} pyrimidin-4-yl) hydrazone-N-allyl-indolyl

Carbaldehyde (8m) IR (KBr) $v_{\max }\left(\mathrm{cm}^{-1}\right): 3342(\mathrm{~N}-$ H), 3080 (aromatic C-H), 2926 (aliphatic C-H), 1602 $(\mathrm{C}=\mathrm{N}), 1513(\mathrm{C}=\mathrm{C})$

${ }^{1} \mathrm{H}$ NMR (300MHz, DMSO- $\left.\mathrm{d}_{6}\right): \delta$ (ppm) 11.15 (bs, $1 \mathrm{H}, \mathrm{NH}) ; 8.58(\mathrm{~s}, 1 \mathrm{H}$, pyrimidinyl); 8.52- $8.45(\mathrm{~m}$, $1 \mathrm{H}, \mathrm{Ar}-\mathrm{H}) ; 7.82-7.76(\mathrm{~m}, 2 \mathrm{H},=\mathrm{CH} \& \mathrm{Ar}-\mathrm{H})$; 7.48$7.46(\mathrm{~d}, J=7.8 \mathrm{~Hz}, 1 \mathrm{H}, \mathrm{Ar}-\mathrm{H}) ; 7.23-7.18$ (m, 2H, Ar$\mathrm{H})$; $6.07-5.97\left(\mathrm{~m}, 1 \mathrm{H},-\mathrm{CH}=\mathrm{CH}_{2}\right) ; 5.20-5.15(\mathrm{~m}, 2 \mathrm{H}$, $\left.\mathrm{CH}=\underline{\mathrm{C}}_{2}\right) ; 4.86-4.77\left(\mathrm{~m}, 2 \mathrm{H}, \mathrm{N}-\mathrm{C}_{2}\right) ; 2.91$ (bs, $2 \mathrm{H}$, $\mathrm{CH}_{2}$ ); 2.72 (bs, $2 \mathrm{H}, \mathrm{CH}_{2}$ ); 1.77 (bs, $4 \mathrm{H}, 2 \times \mathrm{CH}_{2}$ ).

2-(5,6,7,8-tetrahydro[1]benzothieno[2,3d]pyrimidin-4-yl)hydrazone-N-4-nitrobenzylindolyl Carbaldehyde (8n) IR (KBr) $\mathrm{V}_{\max }\left(\mathrm{cm}^{-1}\right)$ : $3372(\mathrm{~N}-\mathrm{H}), 2933$ (aliphatic $\mathrm{C}-\mathrm{H}), 1623(\mathrm{C}=\mathrm{N}), 1576$ $(\mathrm{C}=\mathrm{C}), 1236(\mathrm{C}-\mathrm{N}) .{ }^{1} \mathrm{HNMR}\left(300 \mathrm{MHz}, \mathrm{DMSO}-\mathrm{d}_{6}\right): \delta$ (ppm) $11.16 \quad(\mathrm{bs}, 1 \mathrm{H}, \mathrm{NH}) ; 8.60-8.50 \quad(\mathrm{~m}, \quad 1 \mathrm{H}$, pyrimidinyl and $=\mathrm{CH}) 8.172(\mathrm{~s}, 1 \mathrm{H}, \mathrm{Ar}-\mathrm{H}) ; 8.10(\mathrm{~s}$, 1H, Ar-H); 7.79 (s, 1H, Ar-H); 7.43 (s, 3H, Ar-H); 7.21 (s, 2H, Ar-H); 5.65(s, 2H, N-CH $) ; 2.99$ (s, 2H, $\left.\mathrm{CH}_{2}\right) ; 2.72$ (s, $\left.2 \mathrm{H}, \mathrm{CH}_{2}\right) ; 1.77$ (s, $\left.4 \mathrm{H}, 2 \times \mathrm{CH}_{2}\right)$.

${ }^{13} \mathrm{C} \quad \mathrm{NMR} \quad\left(300 \mathrm{MHz}, \quad \mathrm{DMSO}-d_{6}\right): \quad \delta \quad(\mathrm{ppm}):$ 156.32 ( $\underline{\mathrm{C}}_{4}$ of pyrimidinyl), 150.20 ( $\underline{\mathrm{C}}_{2}$ of pyrimidinyl), 146.52(S-C-N), 144.75( $\mathrm{C}=\mathrm{N}-\mathrm{NH}), \quad 137.93,134.65$, $132.21,130.92,125.23,123.56,122.75,120.99$, 119.50, 112.27, 110.37, $34.68\left(\mathrm{~N}-\underline{C H}_{2}\right), 26.02\left(\underline{\mathrm{C}}_{8}\right)$,

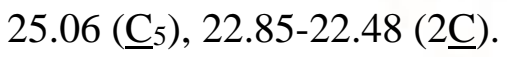

\section{Molecular Modeling}

Molecular docking experiments to investigate the binding modes of synthesized derivatives was done by using the Molecular Design Suite (V-Life MDS 3.0 software package, version 3.0; from Vlife Sciences, Pune, India), on a Windows 7, Windows Server 2008 R2 (operating system version 6.1), Genuine Intel Computer ID: 783232402132454023. 


\section{Docking}

The challenge of the receptor-ligand docking approach is predict the preferred orientation of one molecule to second when bound to each other to form a stable complex and used in virtual screening or lead optimization for drug screening and design [15]. Distinction of good or bad docked conformation is based on scoring or fitness function. MDS uses fitness functions on only electrostatic and both steric and electrostatic interactions between receptor-ligand. There are many scoring functions force such as dock score, Piecewise Linear Pairwise score (PLP), potential of mean force (PMF) score, steric and electrostatic score, etc. Grip Docking incorporates the PLP function that includes ligand-receptor interactions of hydrogen bonding (donor-acceptor), repulsions (donor-donor, acceptor-acceptor) and dispersion (involving non-polar group interactions) types [16]. Structures were sketched using the 2D draw application provided in the main window and then all the SDF and The conformers found with their best score were saved in output folder. The optimized ligands were then tested for numerous interactions of ligand with receptor having hydrogen bonding and other types of other interactions like hydrophobic bonding and van der Waal's interaction. Dock score of compounds are discussed in table 2 and 3.

\section{Antimicrobial activity}

The MIC of synthesized compounds were carried out by broth micro dilution method [17]

\section{Method -Broth micro dilution method}

A polystyrene tray containing 80 wells is filled with small volumes of serial two-fold dilutions of different antibiotics. The inoculum suspension and standardization is done according to McFarland standard. The bacterial inoculum is then inoculated into the wells and incubated at $37^{\circ} \mathrm{C}$ overnight. The lowest concentration of antibiotic that completely inhibits visual growth of bacteria (no turbidity) is recorded as MIC.

\section{Antibacterial activity-Test organism}

Staphylococcus aureus (MTCC 96), Staphylococcus pyrogenes (MTCC 442), Escherichia coli (MTCC 443), Pseudomonas aeruginosa (MTCC 1688)

\section{Antifungal activity-Test organism:}

Candida albicans (MTCC 227),Aspergillus niger (MTCC 282),Aspergillus clavatus (MTCC 1323)
Preparation of sample solution

Serial dilutions of test compounds were prepared. Dimethylsulphoxide (DMSO) was used as solvent. Standard drug: Ampicillin (Anti-bacterial activity) Griseofulvin (Anti-fungal activity)

Media: Mueller Hinton broth and Sabouraud's broth was used as nutrient media to grow for bacteria and fungus respectively. Inoculums size for test strain was adjusted to 106 CFU (Colony Forming Unit per milliliter by comparing the turbidity. [18]

Procedure: Serial dilutions were prepared in primary and secondary screening. The control tube containing no antibiotic was immediately sub cultured (before inoculation) by spreading a loopful evenly over a quarter of plate of medium suitable for the growth of the test organism and put for incubation at $37^{\circ} \mathrm{C}$ for bacteria and $22^{\circ} \mathrm{C}$ for fungus. The tubes were then incubated overnight. The MIC of the control organism was read to check the accuracy of the drug concentrations. The lowest concentration inhibiting growth of the organism was recorded as the MIC. Each test compound was diluted obtaining $500 \mu \mathrm{g} / \mathrm{mL}$ concentration, as a stock solution. In primary screening 10050 and $25 \mu \mathrm{g} / \mathrm{mL}$ concentrations of the test compounds were taken. The active synthesized compounds found in this primary screening were further tested in the second set of dilution against all microorganisms. The drugs found active in primary screening were similarly diluted to obtained 100,50 , $25,012.5, \quad 6.250, \quad 3.125$ and $1,5625 \mu \mathrm{g} / \mathrm{mL}$ concentrations. The highest dilution showing at least 99\% inhibition is taken as MIC.

Results and discussion:

\section{Chemistry}

The required aminothiophene ester (4) was synthesized by using Gewald reaction between cyclohexanone and ethylcyanoacetate by using diethyl amine as a base. The FT-IR spectrum, aminothiophene ester (4) showed the strong characteristic peaks at $1647 \mathrm{~cm}^{-1}$ due to the carbonyl groups of ester. The ester was then cyclized and the product of cyclaization (i.e, 4-hydroxy-5,6tetramethylenethieno[2,3-d]pyrimidine, 5) was subjected to chlorination by using $\mathrm{POCl}_{3}$. The structure of chlorinated product i.e 4-chloro-5,6,7,8tetrahydro-benzothieno[2,3-d]pyrimidine (6) was confirmed by the disappearance of carbonyl peak at $1658 \mathrm{~cm}-1$ and by the presence of $\mathrm{C}-\mathrm{Cl}$ stretching at $832 \mathrm{~cm}-1$. The compound 6 was upon hydrazinolysis 
with hydrazine hydrate afford the required Hydrazinyl-5,6,7,8-tetrahydro-[1]benzothieno[ 2,3d]pyrimidine (7) in a good yield after purification. The formation of 7 was confirmed by the characteristics stretching of N-H group at $3308 \mathrm{~cm}-1$ and peak due to $\mathrm{C}=\mathrm{N}$ stretch was appeared at 1676 $\mathrm{cm}-1$. The ${ }^{1} \mathrm{H}-\mathrm{NMR}$ spectrum of 7 showed the singlet of pyrimidinyl ring at $\delta 8.34$ whereas, appearance of another two singlets at $\delta 7.94$ and $\delta 4.60$ due to the three protons of hydrazine $\left(\mathrm{NH}-\mathrm{NH}_{2}\right)$ moiety confirmed the formation of compound 7.Finally, all the synthesized aldehydes were condensed with 7 in absolute ethanol and few drops of glacial acetic acid to get the various titled compounds i.e 5,6,7,8tetrahydrobenzothieno[2,3-d]pyrimidines hydrazones (8a-n). The purity of all the titled compounds was assessed with TLC by using ethyl acetate and hexane as a mobile phase. Structure of all the newly synthesized compounds was established by using various spectral techniques (FT-IR, ${ }^{1} \mathrm{H}-\mathrm{NMR},{ }^{13} \mathrm{C}-$ NMR. All the titled compounds were obtained in 62$85 \%$.
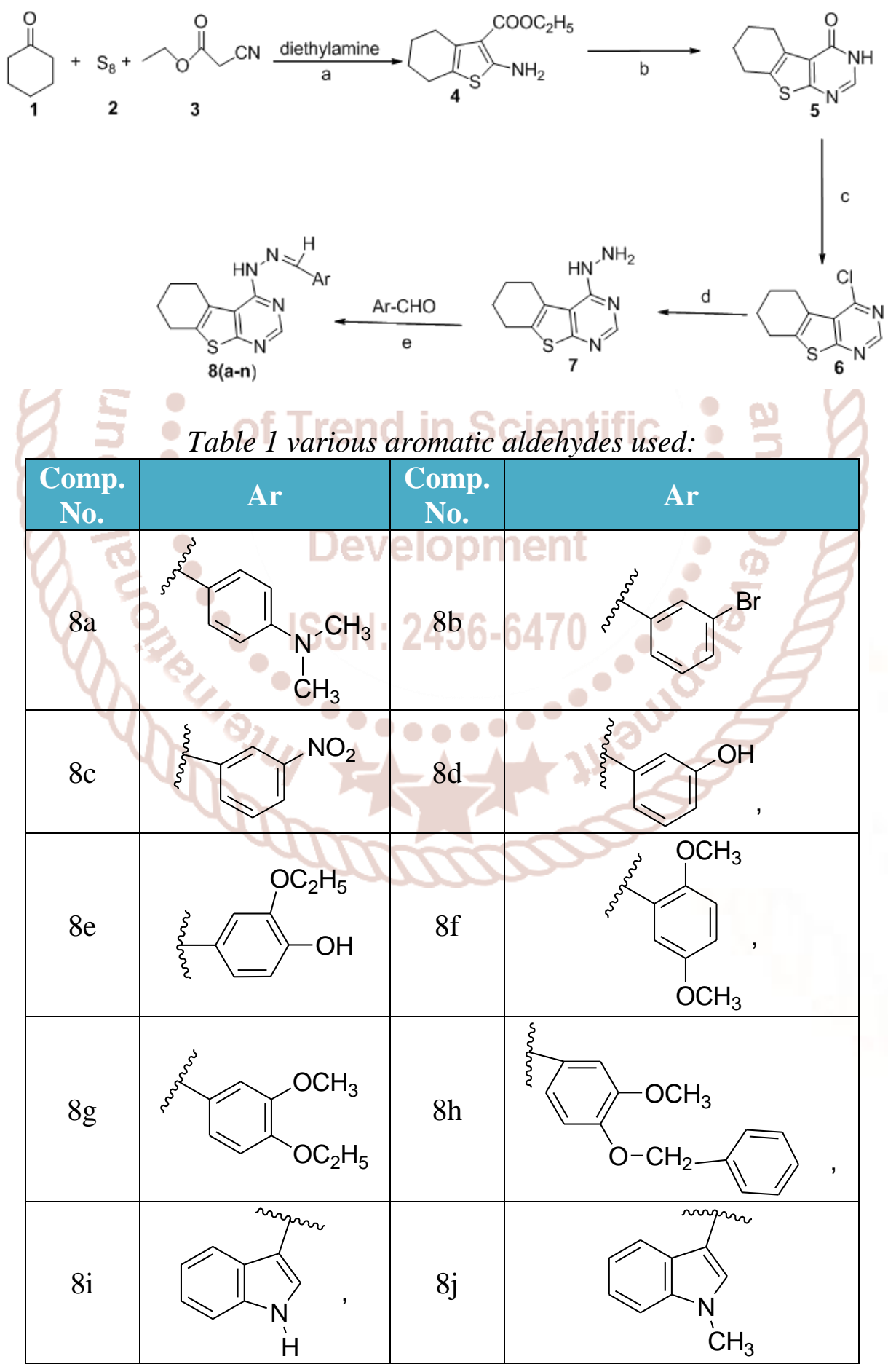


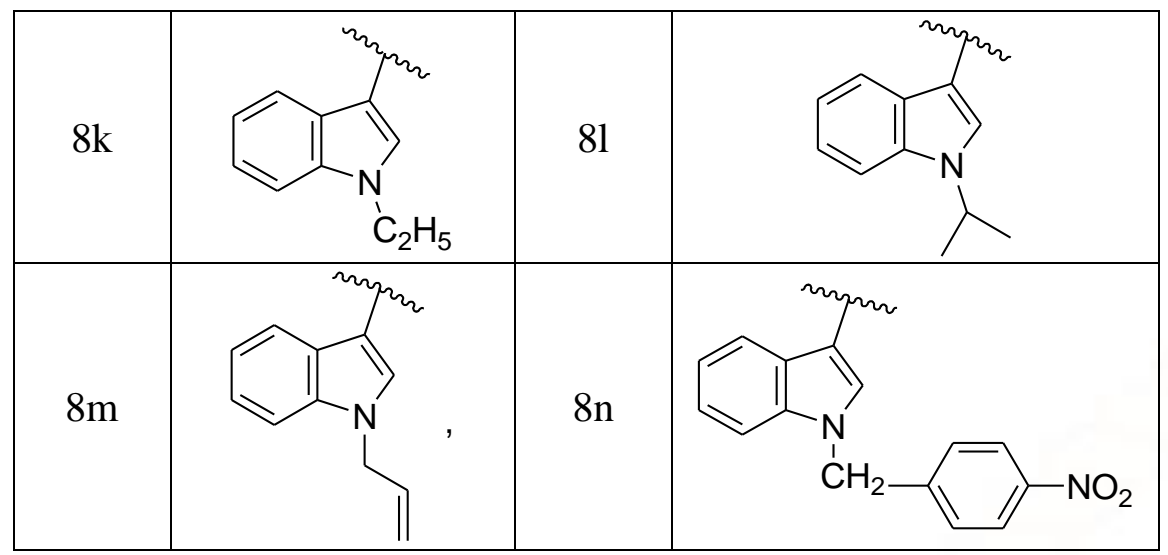

\section{Docking Studies}

Molecular Docking experiment was performed on all 14 derivatives of the series in order to determine the binding affinity with different amino acids as well as to compare the inhibitory activity with reference drugs. Theoretically all the ligand molecules showed encouraging docking scores and interactions of ligands with the receptors show a unique set of bonding such as H-bonding and hydrophobic.-The outcomes of the docking analysis and interactions of ligands with Bacillus subtilus show scores ranging from-58.508112 kcal $/ \mathrm{mol}$ to $-71.187967 \mathrm{ecal} / \mathrm{mol}$, with the best-scored ligand being conformer of compound $8 \mathrm{~h}$. This conformer has shown one hydrogen bonding with HIS180A (2.413938) and have also been proved active compound in series with $20 \mathrm{~mm}$ zone of inhibition. Interaction with
Staphylococcus aureus clears that minimum energy was obtained for compound 8e and 8n with $55.670912 \mathrm{kcal} / \mathrm{mol}$ and maximum energy from $34 \mathrm{kcal} / \mathrm{mol}$ to $40 \mathrm{kcal} / \mathrm{mol}$ was obtained for remaining ligands. Docking results with E.coli indicates that the dock score between the ranges -101.777044 to $77.746887 \mathrm{kcal} / \mathrm{mol}$ have been obtained. The results of docking with enzyme Pseudomonas aeruginosa reveals that all the derivatives have shown good dock scores between the ranges of -92.312649 to$79.515008 \mathrm{kcal} / \mathrm{mol}$. The minimum score have been given by ligand $8 \mathrm{~h}$ with zero hydrogen bonding interactions. Each compound had hydrophobic interaction of carbon atom of methyl and methoxy group with the common residues.

Table-2 Dock scores of target conformer for enzymes Bacillus subtilus, Staphylococcus aureus, Escherichia coli

\begin{tabular}{|c|c|c|c|c|c|c|c|}
\hline \multirow{2}{*}{ Comp No. } & \multirow{2}{*}{$\begin{array}{c}\text { Comp. } \\
\text { Name }\end{array}$} & $\begin{array}{c}\text { Bacillus sulbtilus PDB code } \\
\text { 1I6W }\end{array}$ & $\begin{array}{c}\text { Staphylococcus aureus } \\
\text { PDB Code-1BDD }\end{array}$ & \multicolumn{2}{c|}{$\begin{array}{c}\text { Escherichia coli } \\
\text { PDB Code-2CCZ }\end{array}$} \\
\cline { 3 - 8 } & & Dock Score & H-Bonds & DDDock Score & H-Bonds & Dock Score & H-Bonds \\
\hline 1 & $8 \mathrm{a}$ & -57.256714 & 0 & -4.297931 & 3 & -56.249301 & 2 \\
\hline 2 & $8 \mathrm{~b}$ & -55.765262 & 0 & -38.631030 & 1 & -101.833841 & 3 \\
\hline 3 & $8 \mathrm{c}$ & -55.471749 & 0 & 7.965386 & 1 & -79.845148 & 4 \\
\hline 4 & $8 \mathrm{~d}$ & -57.334851 & 3 & -17.557645 & 4 & -79.751440 & 5 \\
\hline 5 & $8 \mathrm{e}$ & -58.481544 & 1 & 83.547198 & 2 & -79.044625 & 3 \\
\hline 6 & $8 \mathrm{f}$ & -56.646167 & 0 & 26.751494 & 3 & -78.785131 & 4 \\
\hline 7 & $8 \mathrm{~g}$ & -59.476741 & 2 & -35.108466 & 2 & -78.379130 & 3 \\
\hline 8 & $8 \mathrm{~h}$ & -70.626600 & 1 & -35.012141 & 1 & -77.956758 & 2 \\
\hline 9 & $8 \mathrm{i}$ & -60.802969 & 2 & -55.670912 & 2 & -77.951193 & 3 \\
\hline 10 & $8 \mathrm{j}$ & -57.494202 & 1 & -34.839581 & 4 & -77.746887 & 0 \\
\hline 11 & $8 \mathrm{k}$ & -58.047135 & 1 & -39.949067 & 2 & -97.228023 & 2 \\
\hline 12 & $8 \mathrm{l}$ & -61.652294 & 0 & -40.517750 & 1 & -101.777044 & 2 \\
\hline 13 & $8 \mathrm{~m}$ & -63.517307 & 2 & -55.670912 & 4 & -99.851443 & 4 \\
\hline 14 & $8 \mathrm{n}$ & -74.808904 & 2 & -39.949067 & 4 & -97.228023 & 0 \\
\hline
\end{tabular}




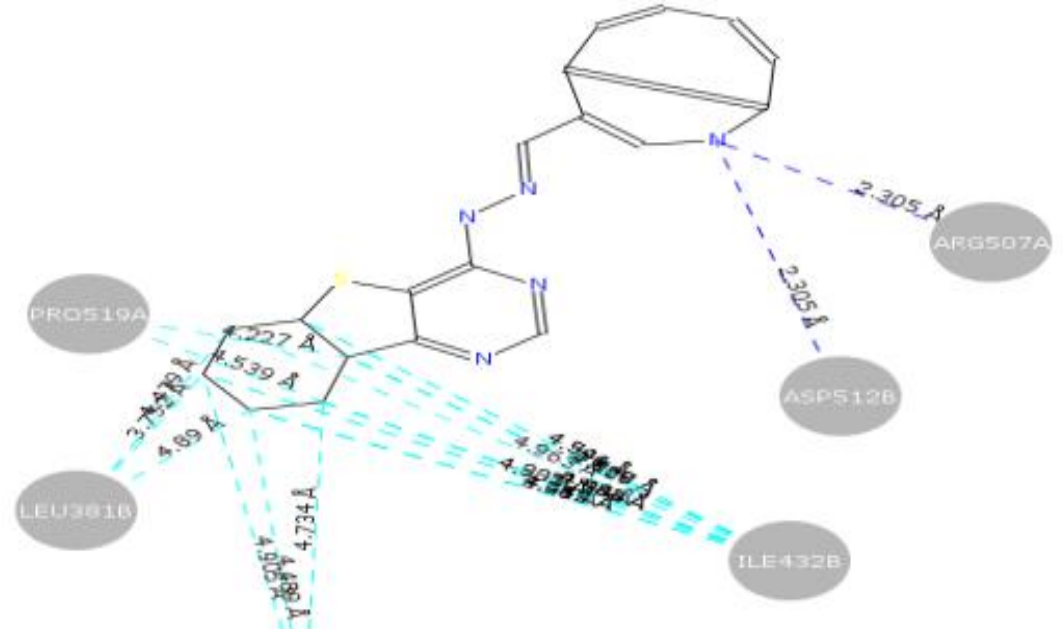

Figure.1 Docking position of synthesized compound 8I in the receptor cavity of Bacillus subtilus with minimum score are represented here with hydrogen bonding interaction and Hydrophobic interaction shown with dotted lines in blue and green colors respectively.

Table 3 Dock scores of target conformer for enzymes Pseudomonas aeruginosa, Candida albicans.

\begin{tabular}{|c|c|c|c|c|c|}
\hline \multirow{2}{*}{ S. No } & \multirow{2}{*}{$\begin{array}{c}\text { compound } \\
\text { name }\end{array}$} & \multicolumn{5}{|c|}{ Pseudomonas aeruginosa } \\
\cline { 3 - 6 } & & Dock Score & H-Bonds & Dock Score & H- Bonds \\
\hline 1 & $8 \mathrm{a}$ & -56.327579 & 0 & -10.167465 & 0 \\
\hline 2 & $8 \mathrm{~b}$ & -57.421525 & 0 & -35.137701 & 0 \\
\hline 3 & $8 \mathrm{c}$ & -57.025621 & 0 & -9.708512 & 0 \\
\hline 4 & $8 \mathrm{~d}$ & -58.848697 & 0 & -21.146097 & 1 \\
\hline 5 & $8 \mathrm{e}$ & -58.010840 & 0 & -25.352467 & 0 \\
\hline 6 & $8 \mathrm{f}$ & -62.754032 & 0 & -6.555559 & 0 \\
\hline 7 & $8 \mathrm{~g}$ & -61.932369 & 0 & -7.880528 & 0 \\
\hline 8 & $8 \mathrm{~h}$ & -74.235698 & 0 & -5.369180 & 1 \\
\hline 9 & $8 \mathrm{i}$ & -58.835544 & 0 & -19.367065 & 2 \\
\hline 10 & $8 \mathrm{j}$ & -63.733447 & 0 & -5.511644 & 0 \\
\hline 11 & $8 \mathrm{k}$ & -62.209991 & 1 & 2.592234 & 1 \\
\hline 12 & 81 & -65.561222 & 0 & 4.460068 & 0 \\
\hline 13 & $8 \mathrm{~m}$ & -65.828413 & 0 & 1.233487 & 0 \\
\hline 14 & $8 \mathrm{n}$ & -68.315483 & 0 & 6.651939 & 0 \\
\hline
\end{tabular}




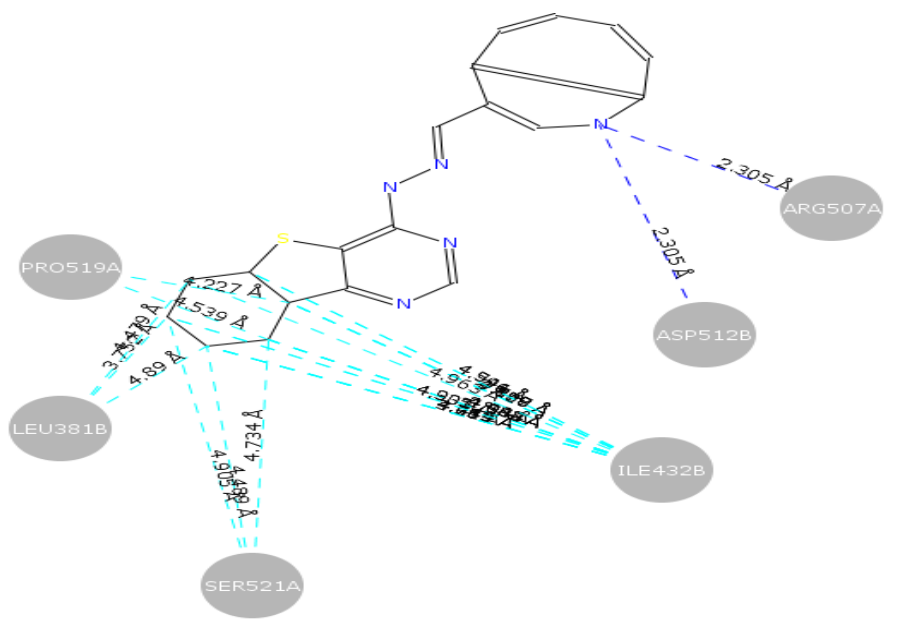

Figure.2 Docking position of synthesized compound 8I in the receptor cavity of Aspergillus niger with minimum score are represented here with hydrogen bonding interaction and Hydrophobic interaction shown with dotted lines in blue and green colors respectively

\section{Antimicrobial Screening:}

The biological activity is a function of physicochemical properties of the targeted molecule and this assessment is made out of chemicals that might fit into an active site. The produced compounds have different pharmacologically active nucleus. All the synthesized compounds were screened for their antimicrobial activity.

Antibacterial activity In vitro antibacterial activity was evaluated against four different strains (Gram positive and Gram negative) using the minimum inhibitory concentration (MIC), which is defined as the lowest concentration at which no visible bacterial growth is observed. Ampicillin was used as positive control. All the synthesized compounds were screened for their antibacterial activity against two Grampositive strains (S. aureus MTCC 96 and S. pyogenus MTCC 442) and two Gram-negative strains (E. coli MTCC 443 and P. aeruginose MTCC 1688). Results of antibacterial evaluation revealed that tested compounds exhibited good activity against Gram positive bacteria. All analogs exhibited potent activity against S. aureus. Compound $\mathbf{8 d}$ was found to be most active against S. aureus having MIC of $3.12 \mu \mathrm{g} / \mathrm{mL}$ which is two-fold greater than standard drug,ciprofloxacin (MIC $=6.25 \mu \mathrm{g} / \mathrm{mL}$ ) whereas, all other compounds also exhibited potent activity against S. aureus with MIC in the range of $3.12-6.25 \mu \mathrm{g} / \mathrm{mL}$. Among various tested analogs 8d, exhibited equipotent activity against gram positive strain $\mathrm{S}$. pyogenus with $\mathrm{MIC}=3.12 \mu \mathrm{g} / \mathrm{mL}$ whereas, compound $8 \mathrm{a}, 8 \mathrm{~d}$ and $8 \mathrm{n}$ also exhibited potent activity against $\mathrm{S}$. pyogenus with $\mathrm{MIC}=3.12-6.25 \mu \mathrm{g} / \mathrm{mL}$ which is equal and slightly less than the standard drug ciprofloxacin $(\mathrm{MIC}=3.12 \mu \mathrm{g} / \mathrm{mL}$ ). All other compounds showed moderate to weak activity.

Few of the tested analogs have showed excellent activity against Gram negative bacteria. Analog 8d exhibited most potent activity against $\mathrm{E}$. coli $(\mathrm{MIC}=$ $1.25 \mu \mathrm{g} / \mathrm{mL}$ ) which is greater than the standard drug ciprofloxacin $(\mathrm{MIC}=3.12 \mu \mathrm{g} / \mathrm{mL})$. Another tested analogs $/ 8 \mathrm{a}, 8 \mathrm{~b}, 8 \mathrm{c}$, and $8 \mathrm{n}$ also showed promising antibacterial activity against E. coli $(\mathrm{MIC}=3.12$ $\mu \mathrm{g} / \mathrm{mL}$ ) which is equal to the ciprofloxacin (3.12 $\mu \mathrm{g} / \mathrm{mL})$. All other analogs showed good to moderate activity $(6.25-12.5 \mu \mathrm{g} / \mathrm{mL})$. Compounds $8 \mathrm{~d}$ exhibited potent activity against $P$. aeruginosa and found to be equipotent with the standard drug ampicillin (MIC = $3.12 \mu \mathrm{g} / \mathrm{mL}$ ). All other compounds exhibited good to moderate activity against $P$. aeruginosa.

\section{Antifungal activity}

Antifungal activity carried out against three different strains (C. albicans, A. niger and A. clavatus) and Griseofulvin was taken as standard drug. Generally, the tested compounds were found to be more active against $C$. albicans as compared to other tested strains. Compound 8d, 8f and 81 showed potent activity against C. albicans with the MIC value of $25.0 \mu \mathrm{g} / \mathrm{mL}$ which is 2-fold greater than the standard drug (MIC = $50.0 \mu \mathrm{g} / \mathrm{mL}$ ). Whereas, compounds $8 \mathrm{a}, 8 \mathrm{e}, 8 \mathrm{~h}, 8 \mathrm{i}$ and $8 \mathrm{k}$ exhibited equipotent activity against $\mathrm{C}$. albicans as compared to standard drug fluconazole. Other analogs were exhibited weak activity against C. albicans. Some of the tested analogs also displayed good activity against $A$. niger although they were less potent as compared to standard used. Compound 8a, $8 \mathrm{~b} 8 \mathrm{c}, 8 \mathrm{~h}, 8 \mathrm{~m}$ and $8 \mathrm{n}$ showed promising activity 
against A. niger with MIC value of $25 \mu \mathrm{g} / \mathrm{mL}$. All other analogs exhibited weak activity or inactive against A. clavatus. From the antimicrobial data it can be concluded that titled compounds were more active against bacteria as compare to fungi. Some of the candidates were found to be active against all tested bacterial strains and displayed broad spectrum of antibacterial profile. Among bacteria, S. aureus and among fungi, E. coli represents the most sensitive bacterial and fungal strain towards various tested analogs.. Few candidates like $8 \mathrm{a}, 8 \mathrm{f}$ and $8 \mathrm{~h}$ were found to be active against bacteria as well as fungi and emerged as broad spectrum of antimicrobial agents.

Table $\mathbf{- 4}$ In vitro antibacterial activity (MIC) values of tested compounds $\mathrm{MIC}(\mu \mathrm{g} / \mathrm{mL})$ and $\mathrm{pMIC}$ value

\begin{tabular}{|c|c|c|c|c|}
\hline & \multicolumn{2}{|c|}{ Gram positive bacteria } & \multicolumn{2}{|c|}{ Gram negative bacteria } \\
\hline \multirow{2}{*}{ Compounds } & $\begin{array}{c}\text { S. aureus } \\
\text { MTCC 96 }\end{array}$ & $\begin{array}{c}\text { S. pyogenus } \\
\text { MTCC 442 }\end{array}$ & $\begin{array}{c}\text { E. coli } \\
\text { MTCC 443 }\end{array}$ & $\begin{array}{c}\text { P. aeruginosa } \\
\text { MTCC 1688 }\end{array}$ \\
\hline $\mathbf{8 a}$ & $\mathbf{6 ( 1 . 7 6 )}$ & $3.12(1.44)$ & $3(2.06)$ & $12.5(1.44)$ \\
\hline $\mathbf{8 b}$ & $12.5(1.49)$ & $25(1.18)$ & $3(2.11)$ & $12.5(1.49)$ \\
\hline $\mathbf{8 c}$ & $25(1.15)$ & $25(1.15)$ & $3(2.07)$ & $25(1.15)$ \\
\hline $\mathbf{8 d}$ & $\mathbf{3 . 1 2}(\mathbf{2 . 0 3})$ & $\mathbf{3 ( 2 . 0 3 )}$ & $\mathbf{1 . 5}$ & $\mathbf{3 ( 2 . 0 3 )}$ \\
\hline $\mathbf{8 e}$ & $12.5(1.46)$ & $12.5(1.46)$ & $6(1.78)$ & $12.5(1.46)$ \\
\hline $\mathbf{8 f}$ & $12.5(1.46)$ & $25(1.16)$ & $6(1.78)$ & $12.5(1.46)$ \\
\hline $\mathbf{8 g}$ & $\mathbf{6 . 2 5 ( 1 . 8 0 )}$ & $25(1.18)$ & $12.5(1.09)$ & $25(1.18)$ \\
\hline $\mathbf{8 h}$ & $\mathbf{6 ( 1 . 7 8 )}$ & $12.5(1.47)$ & $6(1.78)$ & $25(1.16)$ \\
\hline $\mathbf{8 i}$ & $12.5(1.44)$ & $50(0.84)$ & $25(1.14)$ & $25(1.14)$ \\
\hline $\mathbf{8 j}$ & $12.5(1.46)$ & $12.5(1.46)$ & $12.5(1.46)$ & $12.5(1.46)$ \\
\hline $\mathbf{8 k}$ & $25(1.17)$ & $25(1.17)$ & $25(1.17)$ & $25(1.17)$ \\
\hline $\mathbf{8 1}$ & $\mathbf{6 ( 1 . 8 1 )}$ & $12.5(1.49)$ & $25(1.19)$ & $25(1.19)$ \\
\hline $\mathbf{8 m}$ & $12.5(1.49)$ & $25(1.18)$ & $6(1.80)$ & $12.5(1.49)$ \\
\hline $\mathbf{8 n}$ & $\mathbf{6 ( 1 . 9 0 )}$ & $6(1.90)$ & $3(2.14)$ & $100(0.68)$ \\
\hline Ciprofloxacin & $\mathbf{6 ( 1 . 7 4 )}$ & $3(2.04)$ & $3(2.04)$ & $3(2.04)$ \\
\hline & & & & \\
\hline
\end{tabular}

Table-5 In vitro antifungal activity (MIC) values of tested compounds $\mathrm{MIC}(\boldsymbol{\mu g} / \mathbf{m L})$

\begin{tabular}{|c|c|c|c|}
\hline Compounds & $\begin{array}{c}\text { C.albicans } \\
\text { MTCC 227 }\end{array}$ & $\begin{array}{c}\text { A.niger } \\
\text { MTCC 282 }\end{array}$ & $\begin{array}{c}\text { A.clavatus } \\
\text { MTCC 1323 }\end{array}$ \\
\hline $8 \mathrm{a}$ & $50(0.84)$ & $25(1.14)$ & $25(1.14)$ \\
\hline $8 \mathrm{~b}$ & $100(0.58)$ & $25(1.18)$ & $50(0.88)$ \\
\hline $8 \mathrm{c}$ & $100(0.54)$ & $25(1.15)$ & $50(0.84)$ \\
\hline $8 \mathrm{~d}$ & $\mathbf{2 5}(\mathbf{1 . 1 1})$ & $50(0.81)$ & $50(0.81)$ \\
\hline $8 \mathrm{e}$ & $50(0.86)$ & $50(0.86)$ & $50(0.86)$ \\
\hline $8 \mathrm{f}$ & $\mathbf{2 5}(\mathbf{1 . 1 6})$ & $50(0.86)$ & $25(1.16)$ \\
\hline $8 \mathrm{~g}$ & $100(0.58)$ & $50(0.88)$ & $25(1.18)$ \\
\hline $8 \mathrm{~h}$ & $50(0.86)$ & $25(1.70)$ & $50(0.86)$ \\
\hline $8 \mathrm{i}$ & $50(0.84)$ & $25(1.14)$ & $50(0.84)$ \\
\hline $8 \mathrm{j}$ & $100(0.55)$ & $50(0.85)$ & $50(0.85)$ \\
\hline $8 \mathrm{k}$ & $50(0.87)$ & $50(0.87)$ & $25(1.17)$ \\
\hline $8 \mathrm{l}$ & $\mathbf{2 5 ( 1 . 1 9 )}$ & $50(0.89)$ & $25(1.19)$ \\
\hline $8 \mathrm{~m}$ & $100(0.58)$ & $25(1.90)$ & $25(1.90)$ \\
\hline $8 \mathrm{n}$ & $100(0.68)$ & $25(1.28)$ & $50(0.98)$ \\
\hline Fluconazole & $50(0.78)$ & $12.5(1.39)$ & $12.5(1.39)$ \\
\hline
\end{tabular}




\section{Conclusion:}

In conclusion we have Synthesized 14 new tetrahydrobenzothienopyrimidines hydrazones derivatives, determined the physicochemical parameters (melting point), characterized these derivatives by suitable method such as FTIR, ${ }^{1} \mathrm{H}$ NMR, ${ }^{13}$ C-NMR,mass spectroscopy and evaluated newly synthesized derivatives for various biological activities such as antibacterial, antifungal. From the results of antimicrobial evaluation it can be concluded that compounds were most active against bacteria as compared to fungi. Careful examination of structure of various titled compounds revealed that the substituents had variable effect on antimicrobial activity. Many compounds exhibited potent activity against Gram positive bacteria and some of them were also found to exhibited promising activity against Gram negative bacteria. Few of the tested analogs displayed potent antifungal activity. Among Gram positive bacteria, $S$. aureus MTCC 96, Gram negative bacteria $E$. coli MTCC 443 and among fungi, $C$. albicans MTCC 227 represents most sensitive strain against various tested analogs. Some of the candidates $(\mathbf{8 b}, 8 \mathbf{d}, 8 \mathbf{n}, 8 \mathbf{h}, 8 \mathrm{e}$ and $8 \mathrm{c})$ were found to be active against various tested bacterial strains and few of them $(\mathbf{8 a}, \mathbf{8 f}$ and $\mathbf{8 h})$ exhibited broad spectrum of antimicrobial activity and were active against both bacteria as well as fungi.

\section{Acknowledgments:}

The work was supported by Rajendra institute of technology and sciences, Sirsa Haryana and GJUS\&T Hissar, Haryana

\section{References}

1. Hartough HD, Thiophene and its derivatives. The chemistry of heterocyclic compounds. 3, 2009, 114-17.

2. Aponte JC, Abraham J. Vaisberg DC, German G, Estevez Y, Trypanoside, anti-tuberculosis, leishmanicidal, and cytotoxic activities of tetrahydrobenzothienopyrimidines. Bioorg \& Med Chem. 18, 2010, 2880-86.

3. Shrikant B. Kanawade, Raghunath B. Toche, Dhanji P, Synthetic Tactics of New Class of 4Aminothieno [2,3-d]pyrimidine-6-carbonitrile derivatives acting as antimicrobial Agents. Eur J Med Chem, 47 (3), 2013, 673-80.

4. Dewal MB, Wani AS, Vidaillac C, Oupický D, Rybak MJ, Firestine SM, Thieno[2,3-d] pyrimidinedione derivatives as antibacterial agents. Eur J Med Chem. 51, 2012, 145-53.

5. Hayam M, Omaima G, Ola H, Ibrahim M, Ashmawy EI. Synthesis and biological evaluation of thieno[20,30:4,5]pyrimido[1,2-b] $[1,2,4]$ triazines and thieno[2,3-d] $[1,2,4]$ triazolo[ 1,5 -a]pyrimidines as antiinflammatory and analgesic agents. Eur $\mathrm{J}$ Med Chem. 62, 2013, 341-51.

6. Rishipathak D., Shirodkar P., Design and Molecular Docking Studies of Some 1,3,4Oxadiazole Derivatives. Int. J. Pharm. Sci. Rev. Res., 23(1), 2013, 285-289.

7. Horiuchi T, Chiba J, Kouichi U, Tsunehiko S. Synthesis, biological evaluation, and structureactivity relationships and Discovery of novel thieno[2,3-d]pyrimidin-4-yl hydrazone-based inhibitors of Cyclin D1-CDK4;Bioorg \& Med Chem Letters. 19, 2009, 305-08.

8. Pédeboscq S, Gravier D, Casadebaig F, Geneviève H, Gissot A, Christophe R, Ichas F, Giorgi FD, Lartigue L, Pometan JP. Synthesis and evaluation of apoptosis induction of thienopyrimidine compounds on KRAS and BRAF mutated colorectal cancer cell lines. Bioorg \& Med Chem, 20, 2012 6724-6731.

9. Bachwani, M., Kumar R, Molecular Docking: A review. Int J Res Ayurveda Pharm.; 2(6), 2011, 1746-1751.

10. Malleshappa, N.N., Patel, H.M., A comparative QSAR analysis and molecular docking studies of quinazoline derivatives as tyrosine kinase (EGFR) inhibitors: A rational approach to anticancer drug design. Saudi J. Chem. Soc. 17, 2013,361-379.

11. Bhuvaa, H.A., Kini, S. G, Synthesis, anticancer activity and docking of some substituted benzothiazoles as tyrosine kinase inhibitors.J Mol Graph Model. 29, 2010, 32-37.

12. Gouda, H., Torigoe, H., Saito, A., Sato, M., Arata, Y, NMR Study of the Interaction Between the B Domain of Staphylococcal Protein a and the Fc Portion of Immunoglobulin G. Biochemistry. 31, 1992. 9665-9672.

13. Nikulin, A., Stolboushkina, E., Perederina, A., Vassilieva, I., Blaesi, U., Moll, I., Kachalova, G., Yokoyama, S., Vassylyev, D., Garber, M., Nikonov, S, Acta Crystallogr, Sect.D. 61, 2011, 41-146. 
14. Onkara, Kumar, A.S., Kanakaraju, S., Prasanna, B., Pydisetty, Y., Molecular docking studies, Synthesis and Anti-bacterial properties of new mannich bases., Int J Pharm Bio Sci. 4(2), 2013, 2005, $263-270$.

15. Romagnoli R, Baraldi PG, Carrion MD, Lopez OC, Tolomeo M, Grimaudo S, Antonietta DC, Pipitone MR, Balzarini J, Brancale A, Hamel E, Substituted 2-(3,4,5-trimethoxybenzoyl)benzo[b]thiophene derivatives as potent tubulin polymerization inhibitors; Bioorg \& Med Chem. $18,2010,5114-22$.
16. Rishipathak D, Shirodkar P, Design and Molecular Docking Studies of Some 1,3,4Oxadiazole Derivatives. Int. J. Pharm. Sci. Rev. Res., 23(1), 2013, 53, 285-289.

17. Salman, A. S., Mahmoud, N. A., Aziem AA., Mohamed, M.A., Elsisi D.M, Synthesis, Reactions and Antimicrobial Activity of Some New 3Substituted Indole Derivatives, International Journal of Organic Chemistry, 5, 2015, 81-99.

18. Sharshira, E. M., Hamada, N.M.M., Synthesis, Characterization and Antimicrobial Activities of Some Thiazole Derivatives, Amer. J. of Org. Chem. 2(3), 2013, 69-73.

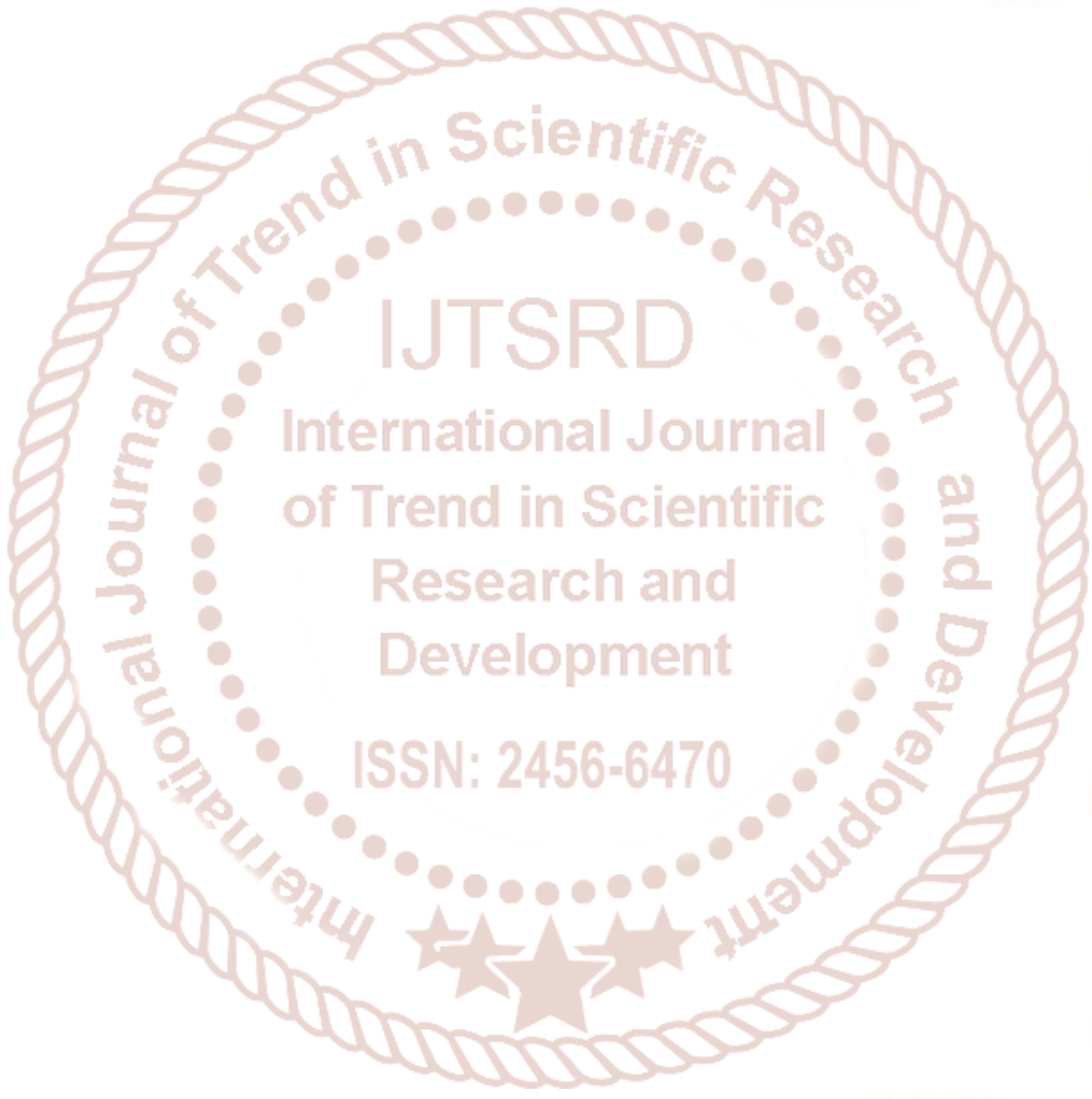

